Ann. Biol. anim. Bioch. Biophys., I962, 2 (3), 209-222.

\title{
RÉGULATION DES SAISONS SEXUELLES CHEZ DES BREBIS DE RACES DIFFÉRENTES AU MOYEN DE DIVERS RYTHMES LUMINEUX
}

\author{
P. MAULÉON ET J. ROUGEOT \\ Station de Recherches de Physiologie animale, \\ Centre national de Recherches zootechniques, Jouy-en-Josas (Seine-et-Oise)
}

SOMMAIRE

Nous avons étudié pendant plusieurs années l'activité sexuelle saisonnière de Brebis Ile-de-France, Texel, Préalpes du Sud et Limousines soumises à deux rythmes lumineux différents : 1) un rythme lumineux annuel reproduisant à peu près le mouvement de variation naturel de la photopériode journalière, l'amplitude de variation sous nos latitudes étant comprise entre 8 et i 6 heures seulement; 2) un rythme lumineux semestriel ne différant du précédent que par sa période, qui réduite de moitié, fut amenée à 6 mois.

En rythme lumineux artificiel annuel, les Brebis ont présenté une seule saison d'activité sexuelle par an pendant la phase des jours courts, comme dans le rythme naturel, et avec les mêmes différences interraciales. Par contre, en rythme lumineux semestriel, les Brebis ont présenté deux phases d'activité sexuelle par an, coïncidant exactement avec les phases des jours croissants et se manifestant même au moment des photopériodes les plus longues.

\section{INTRODUCTION}

On sait, depuis les expériences de YEATES (I949) que les saisons d'activité sexuelle des Brebis sont réglées par le photopériodisme annuel : cet auteur a montré que les Brebis provenant d'un croisement Suffolk $\times$ Border Leicester-Cheviot, entraient en chaleur I4 à I7 semaines après le jour le plus long, et que leurs cycles ostriens disparaissaient au bout du même intervalle de temps après le jour le plus court. Plus tard HAFEZ (I952) montra que la durée de la saison sexuelle chez des Brebis placées dans les mêmes conditions de photopériodisme naturel, à Cambridge, à $52^{\circ}$ de latiture nord, variait suivant l'âge (agnelles, adultes) et l'origine géographique, en altitude et latitude, de la race à laquelle elles appartenaient.

D'après YEATES, l'activité et le repos sexuel sont consécutifs au mouvement même de la variation cyclique de la photopériode : le passage des jours croissants 


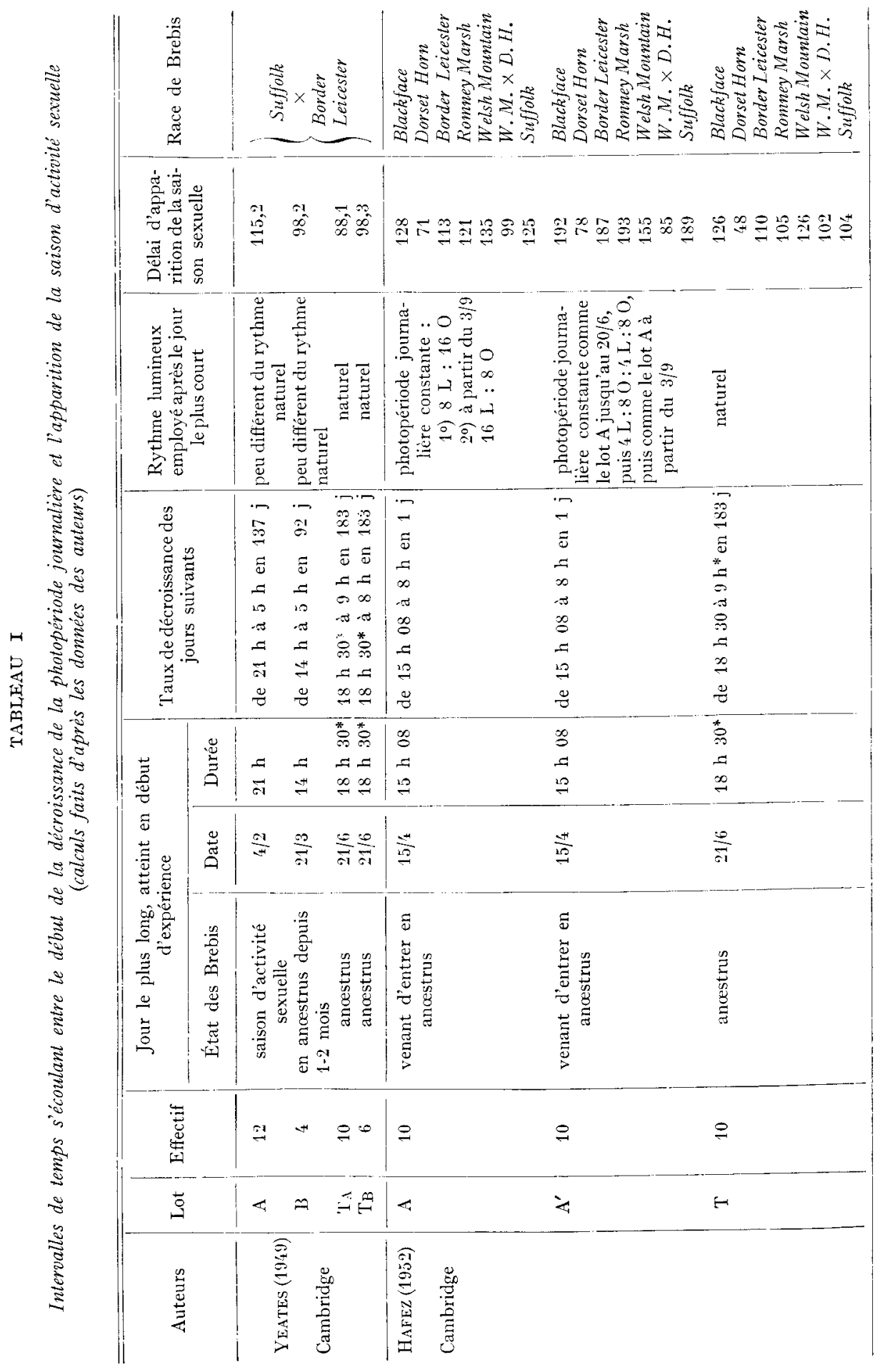



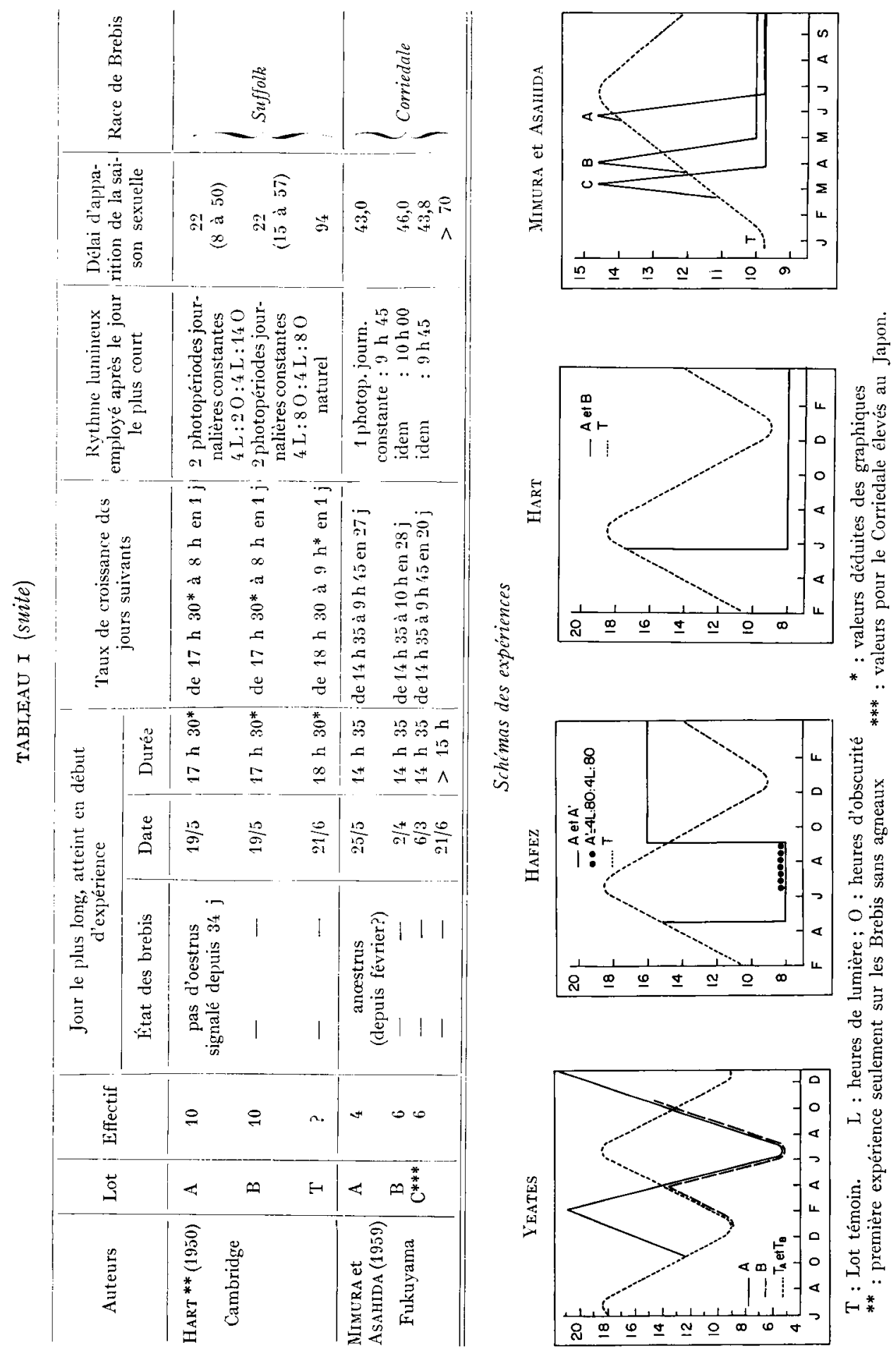
aux jours décroissants induisant, avec un certain délai, les cyles œestriens et le passage inverse entraînant l'anœstrus avec le même délai. Mais HaRT (I952) pense que l'induction et l'arrêt des cycles ne se produisent que pour des photopériodes précises : selon cet auteur les cycles ostriens n'auraient lieu qu'en photopériodes courtes et le mouvement cyclique annuel de la variation de la photopériode ne serait qu'un moyen pour les atteindre (tableau I).

Devant ces interprétations il nous a semblé intéressant d'étudier l'activité sextrelle saisonnière des Brebis lorsqu'on réduisait de moitié, c'est-à-dire de 6 mois, la période de la variation naturelle de la photopériode journalière : en effet, avec cette périodicité, la reprise de croissance des jours s'effectue I 3 semaines après les jours de durée maximale soit sensiblement au moment où, d'après YEATES et HAFEZ, les Brebis commencent généralement à entrer en saison sexuelle.

Nous comparons donc dans cette étude l'activité sexuelle saisonnière, en rythme lumineux annuel et semestriel, de Brebis de races très différentes: Limousine, Préalpes du Sud, Texel, Ile-de-France (tableau 2).

\section{MATÉRIEL ET MÉTHODES}

\section{PROTOCOLE EXPÉRIMENTAL}

Les observations ont été faites du $\mathrm{I}^{\mathrm{er}}$ août 1958 au $3 \mathrm{I}$ décembre I96I sur $\mathrm{I} 8$ Brebis réparties en 2 lots équivalents correspondant aux 2 traitements lumineux utilisés.

Le protocole expérimental exposé dans le tableau 3 comporte quelques anomalies résultant des expériences faites par ailleurs sur la toison (RovgEoT, 1957 et 1961). C'est ainsi que plusieurs Brebis avaient déjà subi un traitement lumineux avant que des observations sur leur activité sexuelle n'aient été effectuées. Pour la même raison des modifications sont intervenues dans les lots en cours d'expérience. : en particulier, nous ne mentionnons que pour mémoire les observations faites sur les Brebis Ile-de-France qui ont été remplacées dès le 23 août 1959 par les Brebis Texel.

\section{Animaux}

Les Brebis Limousines, Préalpes du Sud et Texel naquirent toutes au printemps, tandis que les Brebis Ile-de-France naquirent en automne, comme c'est le cas dans beaucoup d'élevages de cette race. Lorsque l'expérience commença, elles étaient toutes adultes et aucune d'entre elles ne se trouvait ni en gestation ni en lactation ; il en fut ainsi tout au long de l'expérience.

La composition du régime alimentaire des animaux demeura constante pendant toute la durée de l'expérience (foin de pré, pulpe de betterave, farine de luzerne, sel marin) et la ration servie fut toujours la même. Le poids des Brebis, contrôlé tous les mois, ne montra aucune variation importante. Les toisons ne furent jamais tondues à ras, mais taillées à $3 \mathrm{~cm}$ de la peau, tous les ans à la fin du mois de mai, afin d'éviter de trop grandes perturbations dans la thermogenèse.

La détection des chaleurs eut lieu chaque jour à I6 heures en utilisant un Bélier boute-en-train.

\section{TRAITEMENT IUMINEUX}

Les Brebis du lot I furent soumises à un rythme lumineux annuel reproduisant à peu de chose près la variation saisonnière naturelle de la photopériode journalière sous nos latitudes, l'amplitude de variation demeurant comprise entre 8 et 16 heures. Les Brebis du lot II furent soumises à un rythme lumineux semestriel ne différant du précédent que par la période qui fut réduite à 6 mois, c'est-à-dire à la moitié de celle du rythme lumineux annuel. 


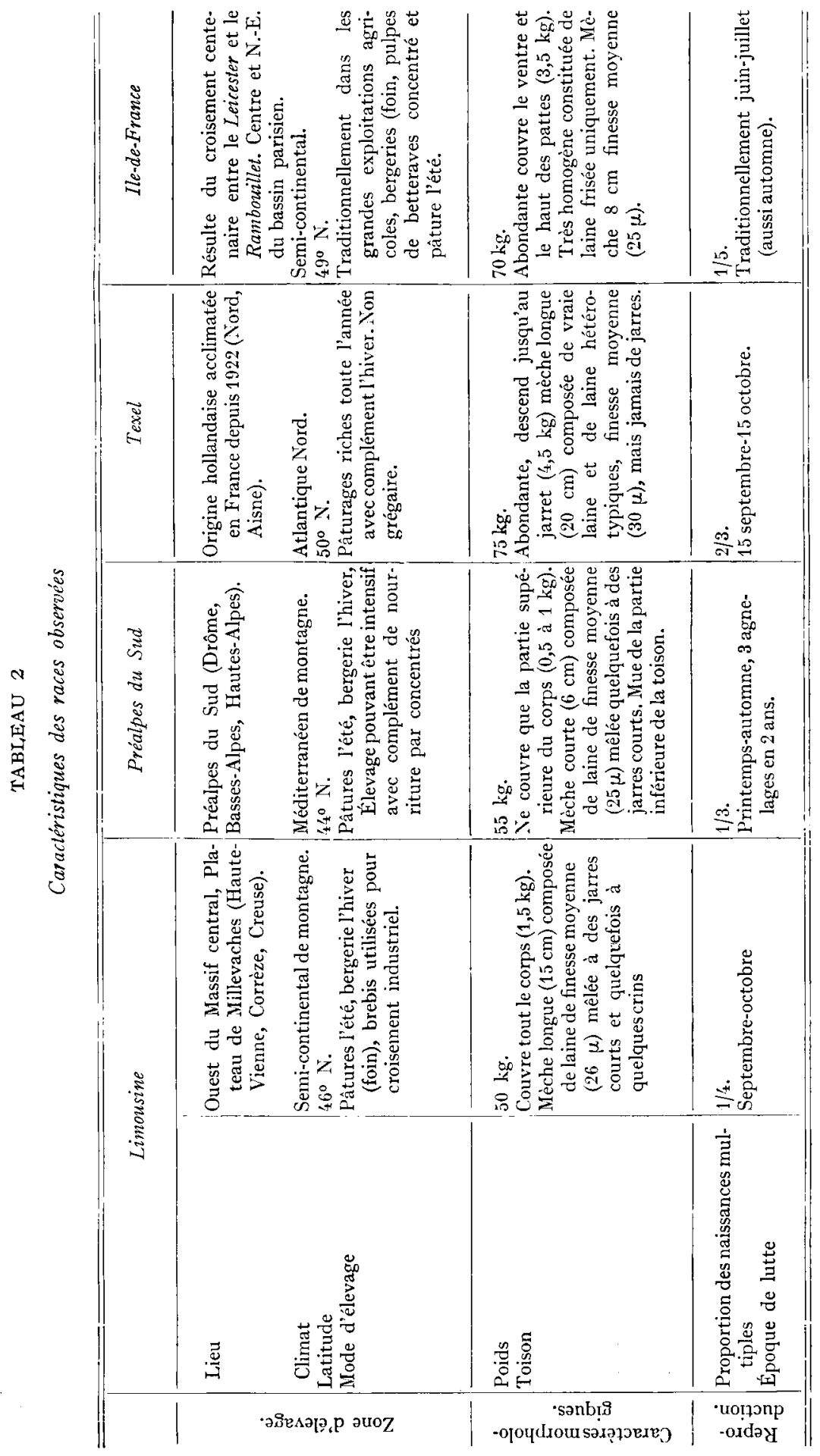


TABI,EAU 3

Constitution des lots de Brebis

\begin{tabular}{|c|c|c|c|c|c|}
\hline \multicolumn{3}{|c|}{$\begin{array}{l}\text { Lot I } \\
\text { Variation anmuelle de la photopériode }\end{array}$} & \multicolumn{3}{|c|}{$\begin{array}{l}\text { Lot II } \\
\text { Variation semestrielle de la photopériode }\end{array}$} \\
\hline $\begin{array}{l}\text { No des } \\
\text { Brebis }\end{array}$ & $\begin{array}{l}\text { Année } \\
\text { Naissance }\end{array}$ & $\begin{array}{l}\text { I'raitement lumineux } \\
\text { avant le } 1 / 8 / 58\end{array}$ & $\begin{array}{l}\text { No des } \\
\text { Brebis }\end{array}$ & $\begin{array}{l}\text { Amméc } \\
\text { Naissance }\end{array}$ & $\begin{array}{l}\text { Traitement lumincux } \\
\text { avant le } 1 / 8 / 58\end{array}$ \\
\hline $\begin{array}{l}\text { I. } 105 \\
\text { I. } 50, k\end{array}$ & $\begin{array}{l}195 \mathbf{1} \\
1955\end{array}$ & $\begin{array}{c}\text { Sen opposition de phase avec } \\
S \text { du lot II du } 21 / 3 / 56 \text { au } \\
21 / 6 / 57, \text { puis } \Lambda\end{array}$ & $\begin{array}{l}\text { L, } 402 \\
\text { L. } 500\end{array}$ & $\begin{array}{l}195 ! \\
1955\end{array}$ & $\begin{array}{c}\text { S sans discontinuer depuis le } \\
21 / 3 / 56\end{array}$ \\
\hline $\begin{array}{ll}\text { L } & 608 \\
\text { L } & 618 \\
\text { P } & 61 ' t \\
\text { P. } & 618\end{array}$ & $\begin{array}{l}1956 \\
1956 \\
1956 \\
1956\end{array}$ & A à partir du $10 / 4 / 58$ & 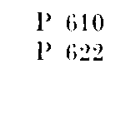 & $\begin{array}{l}1956 \\
1956\end{array}$ & $\mathrm{~S}$ à partir dı $10 / 4 / 5$ \\
\hline $\begin{array}{l}\text { IF } 1430 \\
\text { IF } 1553\end{array}$ & $\begin{array}{l}1950 \\
1950\end{array}$ & $\begin{array}{l}\text { A du } 21 / 3 / 56 \text { au } 23 / 8 / 59 \\
\text { Remplacées par les Texel }\end{array}$ & $\begin{array}{ll}\text { IF } & 1167 \\
\text { IF } & 2239\end{array}$ & $\begin{array}{l}1950 \\
1951\end{array}$ & $\begin{array}{l}\mathrm{S} \text { du } 21 / 3 / 56 \text { au } 23 / 8 / 59 \\
\text { Remplacées par les Texel }\end{array}$ \\
\hline $\begin{array}{l}\mathrm{T} 653 \\
\mathrm{~T} \quad 724\end{array}$ & $\begin{array}{l}1956 \\
1957\end{array}$ & A depuis le $23 / 8 / 59$ & $\begin{array}{l}1738 \\
1766\end{array}$ & $\begin{array}{l}1957 \\
1957\end{array}$ & $\mathrm{~S}$ à partir du $23 / 8 / 59$ \\
\hline
\end{tabular}

$\mathrm{I}$ : : Iimousine ; P : Préalpes du Sud; IF : Ile-de-lirance; T : Texel,

Variation artificielle de la photopériode journalière : $\mathrm{A}:$ annuelle; $\mathrm{S}$ : semestrielle.

En pratique toutes les Brebis furent laissées de 9 h ì i 6 h 30 à la lumière du jour dans un enclos sous abri où elles recevaient en commun leur nourriture. Puis de $\mathrm{i} 6 \mathrm{~h}$ zo à $9 \mathrm{~h}$ le lendemain, chaque lot expérimental fut enfermé dans sa case respective où la lumière du jour ne pouvait pénétrer. Ià, il leur fut attribué un complément de lumière par des tubes fluorescents dont la durée du fonctionnement était fixée par des interrupteurs horaires élcctriques réglés selon le schéma désiré. L'éclairement moyen des cases produit par les tubes fluorescents type lumière du jour fut d'environ 75 lux au niveau des yeux des Brebis.

\section{DÉFINITIONS}

Comme nous ignorons le moment exact où est perçu le stimulus lumineux, nous avons répéré conventionnellement le début et la fin des saisons d'activité sexuelles par rapport au jour le plus long et au jour le plus court de chacun des cycles des rythmes lumineux : nous avons appelé délai d'apparition de la saison d'activité sexuelle, l'intervalle de temps séparant la date du jour le plus long de la date d'apparition du premier ostrus de la saison sexuelle, et celui de cessation de la saison d'activité sexuelle, la date séparant la date du jour le plus court de la date où se produit le dernier ostrus de la saison sexuelle. La durće de la saison d'activité sexuelle est l'intervalle de temps s'écoulant entre le premier oestrus et le dernier oestrus. Une saison sexuelle est caractérisée par une succession non interrompue des cycles cestriens, celle-ci étant considérée comme terminée lorsqu'um intervalle de temps supérieur à deux cycles oestriens, soit 35 jours, s'écoule sans manifestation de chaleur.

\section{RÉSULTATS}

\section{ANALYSE DES GRAPHIQUFS (fig. I)}

En rythme lumineux annuel, la phase d'activité sexuelle a lieu en phase de jours courts, lorsque la photopériode est comprise entre 8 et $\mathrm{I} 2$ heures : elle débute donc au milieu de la phase des jours décroissants et se termine au milieu de la phase des jours croissants. 


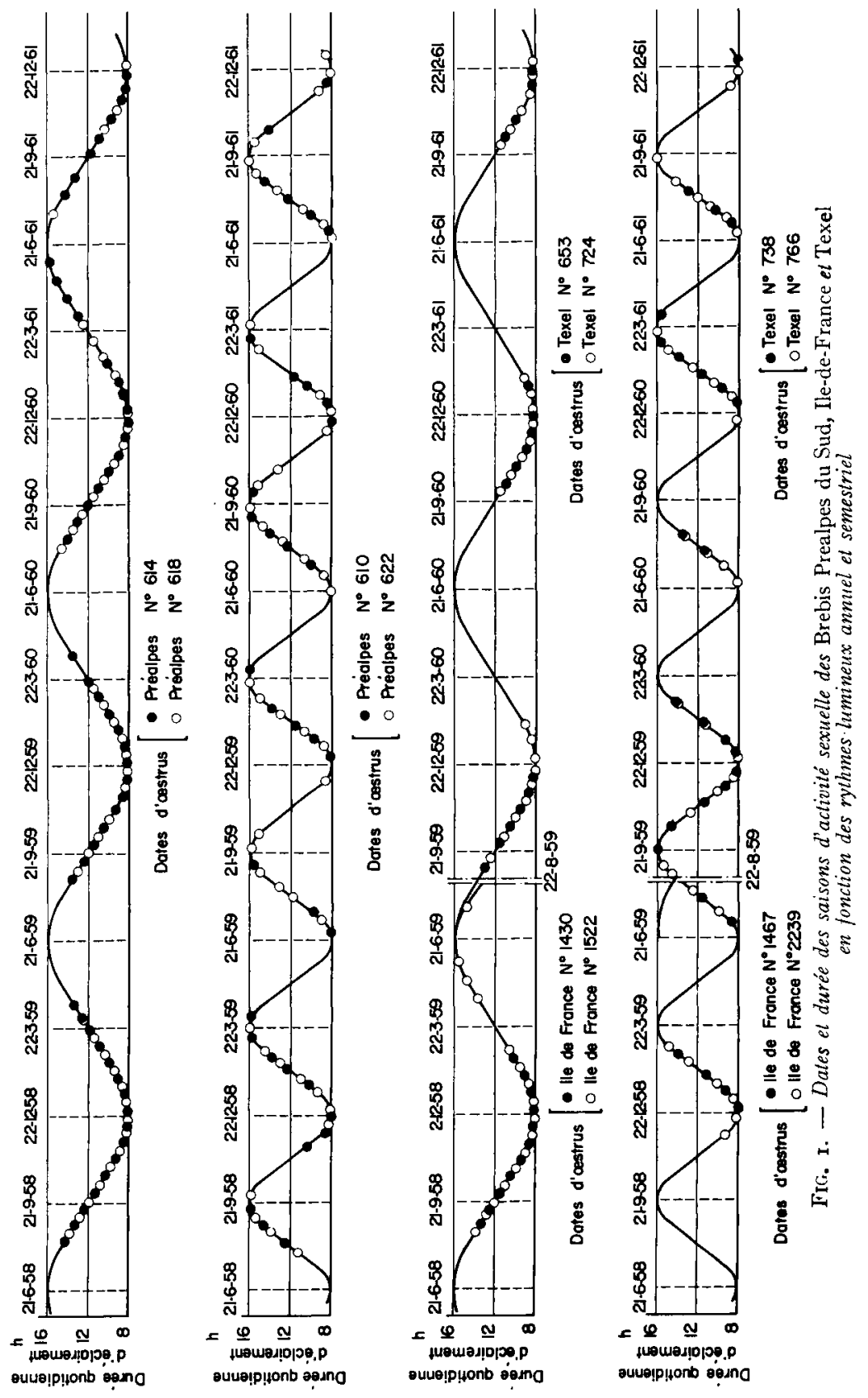




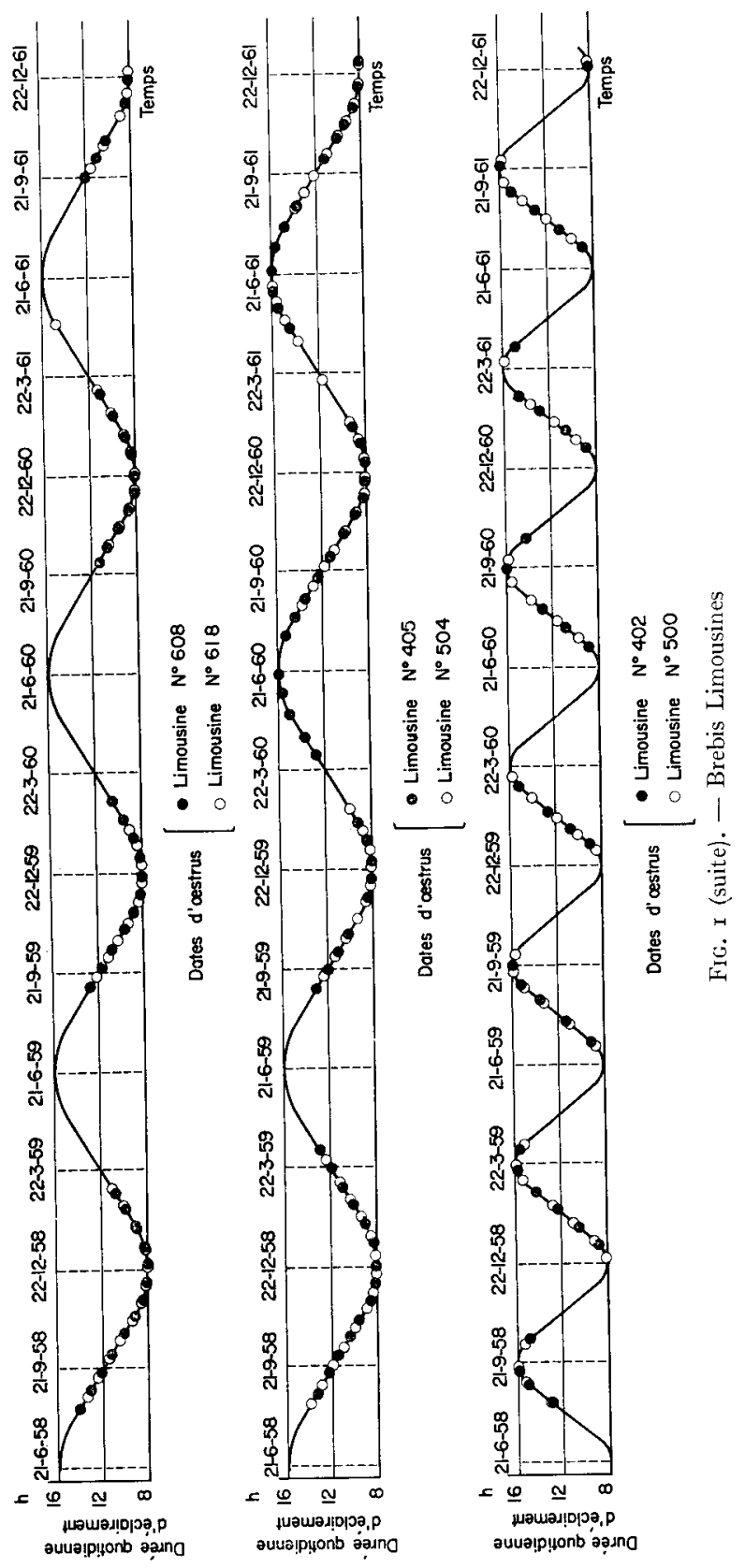


En rythme lumineux semestriel, la phase d'activité sexuelle coïncide exactement avec la phase des jours croissants, y compris le moment où les photopériodes sont les plus longues.

Il en résulte qu'en rythme lumineux semestriel les Brebis présentent deux saisons sexuelles par an dont la durée est sensiblement la moitié de celles qui ont lieu en rythme lumineux annuel.

\section{ANALYSE STATISTIQUE (tableaux 4,5 et 6 ) \\ Io Durée des saisons d'activité sexuelle}

En rythme lumineux annuel, les durées des saisons d'activité sexuelle sont significativement différentes entre les Brebis Limousines, Préalpes du Sud et Texel comme le montrent suffisamment les intervalles de confiance des moyennes.

TABLEAU 4

Variations de la durée des délais d'apparition et de cessation des saisons d'activiti' sexuelle en fonction des parametres des rythmes lumineux

\begin{tabular}{|c|c|c|c|c|c|c|}
\hline \multirow[b]{2}{*}{ Saisons sexuelles } & \multicolumn{3}{|c|}{ Rythme lumineux semestriel } & \multicolumn{3}{|c|}{ Rythme lumineux annuel } \\
\hline & $\mathrm{N}$ & $\overline{\mathrm{X}} \underset{\substack{ \pm \text { jours } \\
\pm s_{\mathrm{X}}}}{(\mathrm{en}}$ & $\begin{array}{l}\text { Intervalle de } \\
\text { confiance }\end{array}$ & $N$ & $\overline{\mathrm{X}} \underset{\text { jours })}{ \pm s_{\mathrm{X}}}(\mathrm{en}$ & $\begin{array}{l}\text { Intervalle de } \\
\text { confiance }\end{array}$ \\
\hline 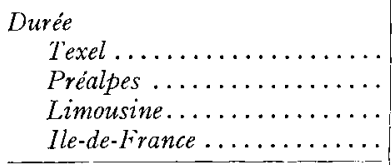 & $\begin{array}{r}6 \\
14 \\
14 \\
2 \\
\end{array}$ & $\begin{array}{l}61,3 \pm 12,54 \\
83,9 \pm 8,35 \\
77,9 \pm 4 \\
86,0\end{array}$ & $\begin{array}{r}29,1-93,5 \\
65,9-101,9 \\
69,0-86,8\end{array}$ & $\begin{array}{c}1 \\
6 \\
10^{*} \\
2\end{array}$ & $\begin{array}{l}112,5 \pm 11,62 \\
216,8 \pm 9,29 \\
171,2 \pm 10,81\end{array}$ & $\begin{array}{l}9,4 \\
0,6 \\
5,6\end{array}$ \\
\hline 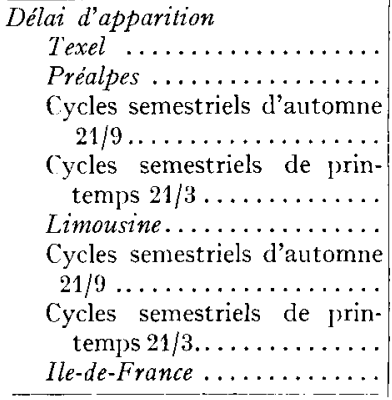 & $\begin{array}{r}8 \\
16\end{array}$ & $\begin{array}{r}99,8 \pm 6,10 \\
97,0 \pm 7,08 \\
76,8 \pm 7,07 \\
117,3 \pm 8,17 \\
112,8 \pm 3,59 \\
104,8 \pm 3,53 \\
120,7 \pm 5,19 \\
100,2\end{array}$ & $\begin{array}{r}85,5-114,1 \\
82,-112,0 \\
60,0-93,4 \\
98,1-136,5 \\
105,2-120,4 \\
96,5-113,1 \\
108,5-132,9\end{array}$ & $\begin{array}{l}6 \\
8\end{array}$ & $\begin{array}{l}97,4 \pm 4,72 \\
66,0 \pm 8,17\end{array}$ & $\begin{array}{l}85,3-109.5 \\
46,8-85,2\end{array}$ \\
\hline 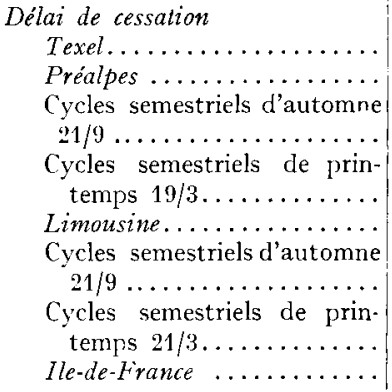 & $\begin{array}{r}8 \\
1 / 4\end{array}$ & $\begin{array}{r}74_{4}, 0 \pm 7,60 \\
94,0 \pm 4,20 \\
92,6 \pm 3,02 \\
96,1 \pm 4,66 \\
101,9 \pm 3,22 \\
96,3 \pm 5,71 \\
101,1 \pm 3,26 \\
88,5\end{array}$ & $\begin{array}{r}52,5-93,5 \\
85,0-103,0 \\
84,9-100,3 \\
85,2-107,0 \\
95,0-108,8 \\
81,7-110,9 \\
93,5-108,7\end{array}$ & $\begin{array}{l}l_{1} \\
6\end{array}$ & $\begin{array}{r}23,0 \pm 13,60 \\
94_{4}, 5 \pm 9,51\end{array}$ & $\begin{array}{l}(-) 20,2-66,2 \\
80,1-118,6\end{array}$ \\
\hline
\end{tabular}

Intervalle de confiance : $m-t_{0,05} \cdot s_{\overline{\mathrm{X}}}<\mu<m+t_{0,05} \cdot s_{\overline{\mathrm{X}}}$.

* I.es résultats de la dernière année pour deux Brebis Limousines ( ${ }^{4} 05$ et 5 ) 1 ) sont exclı $\mathrm{s}$, leur saison sexuelle ayant prisenté des perturbations durant la dernière période. 


\section{TABLEAU 5}

Analyse factorielle de l'influence de la race et de la date du jour le plus long sur le délai d'apparition des saisons sexuelles.

\begin{tabular}{|c|c|c|c|c|}
\hline Source de variation & $\begin{array}{l}\text { Somme des } \\
\text { carrés }\end{array}$ & $\begin{array}{c}\text { Degrés de li- } \\
\text { berté }\end{array}$ & Carré moyen & F \\
\hline Variance totale & 17160 & 31 & & \\
\hline Variance entre traitemeits .. & 9594 & 3 & 3198 & $11,8 * *$ \\
\hline Variance de l'erreur .......... & 7566 & 28 & 270,2 & $\begin{array}{l}\mathrm{F}_{0,05}=2,95 \\
\mathrm{~F}_{0,01}=4,57\end{array}$ \\
\hline \multicolumn{5}{|l|}{$\begin{array}{c}\text { Analyse de la variance entre traite- } \\
\text { ments } \ldots \ldots \ldots \ldots \ldots \ldots \ldots \ldots \ldots \ldots\end{array}$} \\
\hline Saison $\ldots \ldots \ldots \ldots \ldots \ldots \ldots$ & 6384 & 1 & 6384 & $23,6^{* *}$ \\
\hline Race $\ldots \ldots \ldots \ldots \ldots \ldots \ldots \ldots$ & 1984 & 1 & 1984 & $7,3^{\prime} t^{*}$ \\
\hline 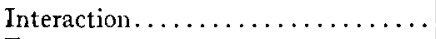 & 1226 & 1 & 1226 & $4,53^{*}$ \\
\hline \multirow[t]{2}{*}{ 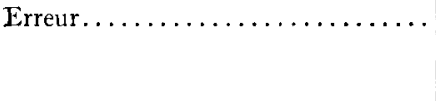 } & 7566 & 28 & 270,2 & \\
\hline & & & & $\begin{array}{l}F_{0,05}=4,20 \\
F_{0,01}=7,64\end{array}$ \\
\hline
\end{tabular}

Test de Tukey sur les différences de moyennes des délais d'apparition des saisons sexuelles entre : Brebis Préalpes "Automne "- Brebis Préalpes "Printemps " $=40,5 * *$

Brebis Limousine "Automne " - Brebis Limousine "Printemps " = 15,9 (N. S.)

Brebis Préalpes "Automne " Brebis Limousine "Automne" $=28,0^{* *}$

Brebis Préalpes "Printemps " - Brebis Limousine "Automne" $=3,4$ (N.S.).

\section{TABLEAU 6}

Analyse de variance de l'infuence du rythme lumineux et de la date du jour le plus long sur le délai de cessation des saisons sexuelles entre Brebis de même race.

\begin{tabular}{|c|c|c|c|c|c|c|c|c|}
\hline \multirow{2}{*}{ Source de Variation } & \multicolumn{4}{|c|}{ Race Préalpes } & \multicolumn{4}{|c|}{ Race Limousine } \\
\hline & $\Sigma x^{2}$ & D.L. & $\begin{array}{l}\text { Carré } \\
\text { moyen }\end{array}$ & $\mathbf{F}$ & $\sum x^{2}$ & D.L. & $\begin{array}{l}\text { Carré } \\
\text { moyen }\end{array}$ & $\mathbf{F}$ \\
\hline $\begin{array}{l}\text { Variance totale } \ldots \ldots \ldots \ldots \ldots \\
\text { Variance traitement........... } \\
\text { Variance de l'erreur......... }\end{array}$ & $\begin{array}{r}4256 \\
41 \\
4215\end{array}$ & $\begin{array}{r}19 \\
2 \\
17\end{array}$ & $\begin{array}{r}20,5 \\
2 \div 7\end{array}$ & $0,08 \mathrm{NS}$ & $\begin{array}{r}12223 \\
6807 \\
5416\end{array}$ & $\begin{array}{r}23 \\
2 \\
21\end{array}$ & $\begin{array}{r}3403 \\
257\end{array}$ & $\begin{array}{c}13,2^{* *} \\
\mathrm{~F}_{0,01}=5,78\end{array}$ \\
\hline $\begin{array}{l}\text { Variance entre rythme annuel } \\
\text { et rythme semestriel......... } \\
\text { Variance entre période de prin. } \\
\text { temps et période d'automne } \\
\text { en rythme semestriel ....... } \\
\text { Variance de l'erreur.......... }\end{array}$ & & & & & $\begin{array}{r}328 \\
5416\end{array}$ & $\begin{array}{r}1 \\
21\end{array}$ & $\begin{array}{l}328 \\
257\end{array}$ & $\begin{array}{c}25,2^{* *} \\
1,3 \mathrm{NS} \\
\mathrm{F}_{0,01}=8,02\end{array}$ \\
\hline
\end{tabular}

En rythme lumineux semestriel, les durées d'activité sexuelle des Brebis des trois races se classent dans le même ordre qu'en rythme lumineux annuel, mais la différence entre les moyennes des durées d'activité sexuelle des Brebis Limousines et celles des Préalpes $d u$ Sud n'est plus significative. 


\section{$2^{\circ}$ Délais d'apparition des saisons d'activité sexuelle}

Chez les Brebis Limousines et Préalpes $d u$ Sud les délais d'apparition des saisons d'activité sexuelle sont significativement plus longs ( I 2 2,8 $\pm 3,59$ et 97,0 $\pm 7,08$ jours) en rythme lumineux semestriel qu'en rythme lumineux annuel $(79,8 \pm 5, \mathrm{I} 6$ et $66,0 \pm 8, \mathrm{I} 7$ jours). On retrouve chez les Brebis Texel une différence de même sens entre les délais d'apparition des saisons d'activité sexuelle qui ont lieu en rythme lumineux semestriel (99,8 \pm 6 , Io jours) et ceux qui ont lieu en rythme lumineux annuel ( $97,4 \pm 4,72$ jours), bien que ces derniers délais soient relativement longs. Mais cette différence est fort réduite et on peut même avancer qu'elle n'est pas significative, bien qu'il ne soit pas possible de le vérifier par un test statistique.

Enfin, en rythme lumineux semestriel, il existe chez les Brebis Préalpes $d u$ Sud uniquement, une différence significative entre les délais d'apparition d'activité sexuelle qui ont lieu au printemps et ceux qui ont lieu en automne.

\section{$3^{\circ}$ Délais de cessation des saisons d'activité sexuelle}

Ils sont significativement plus longs (IOI,9 $\pm 3,22$ jours) en rythme lumineux semestriel qu'en rythme lumineux annuel $\left(68,6 \pm 6,5^{2}\right.$ jours) chez les Brebis Limousines. Il en est sans doute de même pour les Brebis Texel mais pas chez les Brebis Préalpes $d u$ Sud où les délais de cessation d'activité sexuelle ne présentent aucune différence significative selon les rythmes lumineux. Aucune des races étudiées n'a montré de différences significatives, en rythme lumineux semestriel, entre les délais de cessation des saisons sexuelles qui ont lieu au printemps et ceux qui ont lieu en automne.

\section{DISCUSSION}

Le début et la fin des saisons sexuelles, qui se succèdent chez les Brebis soumises aux deux rythmes lumineux utilisés, sont donc étroitement liés aux phases des variations quasi sinusoïdales de la photopériode journalière.

En rythme lumineux annuel nous retrouvons les résultats de YEATES (1949) et de HAFEZ (I952). En ce qui concerne les races ovines françaises on peut simplement souligner que les Brebis Texel dont l'origine est la plus septentrionale ont les saisons sexuelles les plus courtes et que les Brebis Préalpes $d u$ Sud dont l'origine est la plus mériodionale ont les saisons sexuelles les plus longues.

En rythme lumineux semestriel les saisons sexuelles sont réduites de moitié et ont lieu deux fois par an : on peut donc modifier profondément l'activité sexuelle saisonnière des Brebis par le photopériodisme. Mais cette souplesse d'adaptation de l'activité saisonnière sexuelle au rythme lumineux semestriel est limitée par le temps de latence relativement long s'écoulant entre le moment où agit le stimulus lumineux et la manifestation du premier œestrus : étant donnée la rapidité de la variation cyclique de la photopériode en rythme lumineux semestriel, les saisons d'activité sexuelle ne peuvent commencer, lorsque les Brebis sont soumises à ce rythme, qu'au moment où les jours ont atteint leur durée minimale et ne peuvent donc avoir lieu qu'en phase de jours croissants. Pour la même raison, l'arrivée précoce des 
photopériodes longues, en rythme lumineux semestriel, abrégerait la durée de la saison d'activité sexuelle qui ne s'arrête qu'au moment des jours longs.

Mais ceci n'est qu'une interprétation encore incertaine en raison même de 1a nature du critère utilisé pour étudier l'action du photopériodisme sur l'activité sexuelle saisonnière. En effet, l'œestrus est la résultante de l'action des hormones de l'hypophyse sur l'ovaire, des hormones sexuelles sur le système nerveux central et vraisemblablement de nombreux autres relais hormonaux et nerveux.

D'ailleurs les quelques temarques qui nous restent à faire ne font que confirmer la complexité du problème :

Io Si l'on suppose que le stimulus lumineux perçu par les Brebis consiste en une photopériode de durée déterminée, il est nécessairement plus proche du jour de durée maximale d'illumination en rythme lumineux semestriel qu'en rythme lumineux annuel. Aussi, même si l'intervalle de temps stimulus lumineux-premier cestrus est le même dans les deux rythmes lumineux, les "délais d'apparition des saisons d'activité sextuelle ", tels que nous avons définis par rapport au jour le plus long, peuvent ne pas être les mêmes dans les deux cas.

$2^{\circ}$ L'effet du changement de rythme lumineux n'est pas toujours immédiat, la réponse de l'effecteur, tant au point de vue délai que durée, dépendant du passé lumineux et de l'état physiologique (oestrus, ancstrus plus ou moins ancien) des Brebis au moment du changement de rythme (YEATES, x949; HART, I950; HAFEZ I952). C'est ce qui semble s'être produit pour les Brebis Texel no 738 et 766 qui ont subi un changement de rythme lumineux en cours d'expérience : mises en rythme lumineux semestriel le 23 août r958, soit de ux mois après le jour le plus long de l'année, elles présentèrent une saison d'activité sexuelle de durée normale, coïncidant avec celle des deux autres Brebis Texel maintenues en rythme lumineux annuel. En particulier les cycles cestriens de ces deux Brebis eurent lieu pendant la première phase des jours décroissants du rythme lumineux semestriel et il fallut attendre le second cycle de ce rythme lumineux pour que l'activité sexuelle se manifestât uniquement pendant la phase des jours croissants.

Il est donc très difficile de comparer nos résultats en rythme lumineux semestriel, bien que la variation de la photopériode y soit rapide, avec ceux de HART (I950) de Mimura et Asamida (I959), qui ont obtenu des délais d'apparition de saison d'activité sexuelle de quelques semaines en faisant passer instantanément ou très rapidement des Brebis en ancestrus des photopériodes longues aux photopériodes courtes : les races utilisées, l'état physiologique des anjmaux au moment du jour le plus long ainsi que le rythme lumineux étaient fort différents.

$3^{\circ}$ En rythme lumineux semestriel, on a pu montrer chez les Brebis Préalpes $d u S u d$, où l'on a eu suffisamment de données pour faire un test statistique, qu'i1 existait des différences significatives entre les saisons sexuelles de printemps et d'automne. Ces différences peuvent êtres dues à l'action d'un autre facteur saisonnier dont on n'a pu éliminer complètement 1'action (thermopériodisme); il est certain que la sensibilité des structures nerveuses du cerveau qui sont liées au comportement d'œestrus subit une variation saisonnière indépendante de la lumière (RFARDon et RoBINSON, I96I). HAFEZ (I962) a noté des retours d'activité sexuelle chez les Brebis maintenues plusieurs mois en photopériodes journalières constantes longues. Enfin DAUZIER et MAUlEoN (fig. 2) ont observé des cycles d'activité sexuelle chez des Brebis Ile-de-France maintenues pendant 3 ans en lumière permanente : ces Brebis 
sont entrées en anœestrus plus tôt que les témoins de la première année. Puis chaque année les saisons sexuelles sont toujours apparues à la même époque, en automne, mais avec des durées qui diminuaient à chaque fois d'une quantité proportionnelle à la durée initiale. En Australie, RADFord (I96I) fit des observations semblables chez les Brebis Mérinos maintenues en lumière permanente pendant 3 ans. Mais les

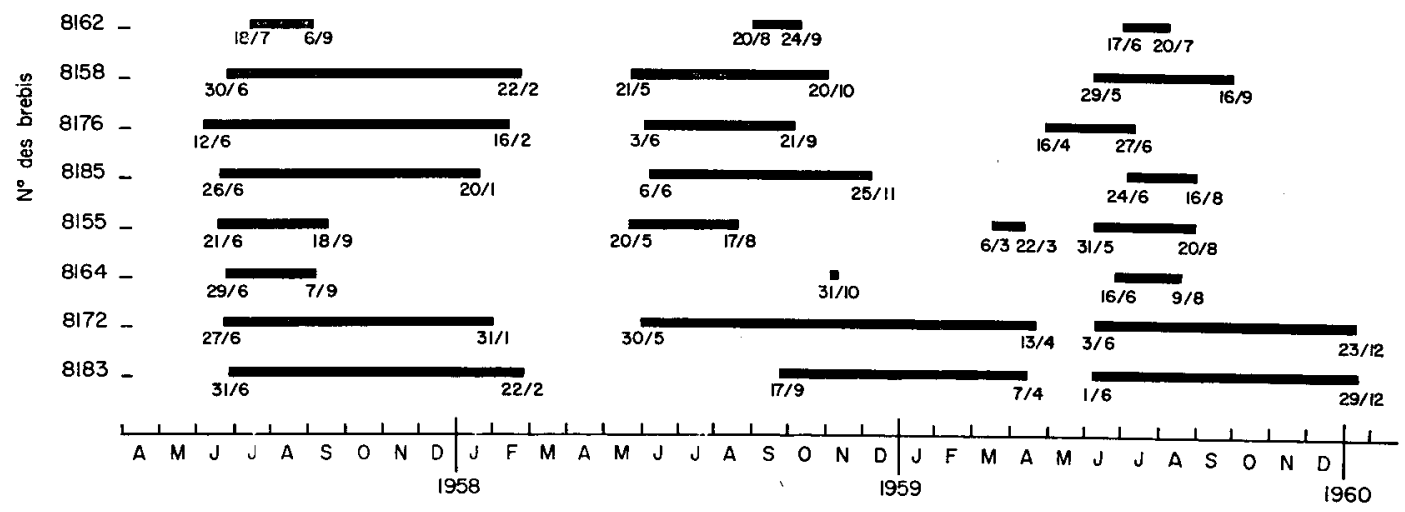

Période pendant laquelle les cycles œestriens se succèdent régulièrement.

FIG. 2. - Saisons d'activité sexuelle chez les Brebis lle-de-France soumises à une illumination permanente pendant 3 ans (Dauzier et Mauleon; début de l'expérience 28-4-58, fin de l'expérience 15-1-61)

rapports des durées des saisons d'activité sexuelle des différents animaux entre eux ne se reproduisirent pas d'une année à l'autre. En outre, si les saisons sexuelles des deux dernières années purent être définies aussi bien à partir de la manifestation des chaleurs que par la présence d'ovulation, il n'en fut pas de même pour la première année où les chaleurs furent rares et où, seule la présence d'ovulation permit de dire que les Brebis Mérinos étaient en activité sexuelle.

Reçu pour publi:ation en juin $\mathbf{1 9 6 2 .}$

\section{SUMMARY}

REGULATION OF SEASONAL SEXUAL ACTIVITY IN DIFFERENT BREEDS OF SHEEP USING DIFFERENT LIGHT RHYTHMS.

I 8 Ile-de-France, Texel, Prealpes du Sud and Limousine ewes were subjected for several years to the following two different artificial light regimes in a study of seasonal sexual activity : I) An annual light rhythm with approximately the same light/dark relationships as is normal for our latitude, i. e., with a variation of amplitude between 8 and I 6 hours. 2) A bi-annual light rhythm in which the same light/dark relationships were condensed into 6 months to give two complete photoperiodic cycles per year.

Sexual activity of all ewes subjected to the annual rhythm was confined to one period of the year, spaced approximately evenly about the shortest days, as occurs with the same breeds in the natural state. Ewes subjected to the bi-annual rhythm exhibited two periods of sexual activity each year, coincident with the periods of increasing light and actually exhibited cycles at the time of longest light hours (fig. T.). 
This difference is thought to be due to the interval between the photoperiodic stimulus and the initiation of cycles, coupled with a shortening of the period of sexual activity by increasing light hours.

Comparison of the sexual seasons of the ewes subjected to these light regimes does not clarify the nature of photoperiodic action, wich appears to be complex.

There were breed $x$ environment interactions. Thus the interval from longest day to first oestrus is longer in the bi-annual light rhythm than in the annual for the Prealpes $d u$ Sud and Limousine ewes, whereas it is the same for the Texel, wich characteristically have short periods of sexual activity. Also, " autumn ", periods of sexual activity begin sooner than the "spring "periods for the Prealpes du Sud ewes, but not for the other breeds.

\section{RÉFÉRENCES BIBLIOGRAPHIQUES}

Dauzier L., Mauleon P. Communications personnelles.

Hafez E. S. E., 1952. Studies on the breeding season and reproduction or the ewe. J. Agric. Sci., 42, 189265.

Hart D. S., 1950. Photoperiodicity in Suffolk sheep. J. Agric. Sci., 42, 293-303.

Mimura K., Asahida Y., 1959. The environmental factors on the lamb growth analytically studied with extra seasonal lambs. I. Extra seasonal production of lambs by artificial light treatments. J. Fac. Fish. anim. Husb., 2, 365-374.

Radford H. M., r96r. Photoperiodism and sexual activity in Merino ewes. Aust. J. Agric. Res., 12, r39I46.

REARDon F. T., Robinson T. J., r96r. Seasonal variation in the reactivity to oestrogen of the ovariectomized ewe. Aust. J. Agric. Res., 12, 320-326.

Rovgeot J., i957. Action de la variation saisonnière de la durée quotidienne d'éclairement sur la mue de certaines fibres de la toison de la race ovine Limousine, C. R. Soc. Biol., 51, 834-837.

RougeOT J., I96r. Action comparée des variations périodiques annuelles et semestrielles de la durée quotidiennes de l'éclairement sur les cycles des follicules des jarres courts de la toison des Brebis Limousines. Relations avec leur cycle de reproduction. Ann. Biol. anim. Bioch. Biophys., 1, 385-402.

YEATES N. T. M., I 949. The breeding season of the ewe with particular reference ot its modification by artificial means using light. J. Agric. Sci., 39, 1-43. 